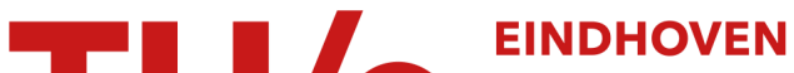 \\ UNIVERSITY OF \\ TECHNOLOGY
}

\section{Superfluidity and spin superfluidity in spinor Bose gases}

Citation for published version (APA):

Armaitis, J., \& Duine, R. A. (2017). Superfluidity and spin superfluidity in spinor Bose gases. Physical Review A, 95(5), 1-10. [053607]. https://doi.org/10.1103/PhysRevA.95.053607

DOI:

10.1103/PhysRevA.95.053607

Document status and date:

Published: 04/05/2017

\section{Document Version:}

Publisher's PDF, also known as Version of Record (includes final page, issue and volume numbers)

\section{Please check the document version of this publication:}

- A submitted manuscript is the version of the article upon submission and before peer-review. There can be important differences between the submitted version and the official published version of record. People interested in the research are advised to contact the author for the final version of the publication, or visit the $\mathrm{DOI}$ to the publisher's website.

- The final author version and the galley proof are versions of the publication after peer review.

- The final published version features the final layout of the paper including the volume, issue and page numbers.

Link to publication

\section{General rights}

Copyright and moral rights for the publications made accessible in the public portal are retained by the authors and/or other copyright owners and it is a condition of accessing publications that users recognise and abide by the legal requirements associated with these rights.

- Users may download and print one copy of any publication from the public portal for the purpose of private study or research.

- You may not further distribute the material or use it for any profit-making activity or commercial gain

- You may freely distribute the URL identifying the publication in the public portal.

If the publication is distributed under the terms of Article $25 f a$ of the Dutch Copyright Act, indicated by the "Taverne" license above, please follow below link for the End User Agreement:

www.tue.nl/taverne

Take down policy

If you believe that this document breaches copyright please contact us at:

openaccess@tue.nl

providing details and we will investigate your claim. 


\title{
Superfluidity and spin superfluidity in spinor Bose gases
}

\author{
J. Armaitis* \\ Institute of Theoretical Physics and Astronomy, Vilnius University, Sauletekio Ave. 3, LT-10222 Vilnius, Lithuania
}

\author{
R. A. Duine \\ Institute for Theoretical Physics and Center for Extreme Matter and Emergent Phenomena, Utrecht University, \\ Princetonplein 5, 3584 CC Utrecht, The Netherlands \\ and Department of Applied Physics, Eindhoven University of Technology, P.O. Box 513, 5600 MB Eindhoven, The Netherlands
}

(Received 14 March 2016; published 4 May 2017)

\begin{abstract}
We show that spinor Bose gases subject to a quadratic Zeeman effect exhibit coexisting superfluidity and spin superfluidity, and study the interplay between these two distinct types of superfluidity. To illustrate that the basic principles governing these two types of superfluidity are the same, we describe the magnetization and particle-density dynamics in a single hydrodynamic framework. In this description spin and mass supercurrents are driven by their respective chemical potential gradients. As an application, we propose an experimentally accessible stationary state, where the two types of supercurrents counterflow and cancel each other, thus resulting in no mass transport. Furthermore, we propose a straightforward setup to probe spin superfluidity by measuring the in-plane magnetization angle of the whole cloud of atoms. We verify the robustness of these findings by evaluating the four-magnon collision time, and find that the time scale for coherent (superfluid) dynamics is separated from that of the slower incoherent dynamics by one order of magnitude. Comparing the atom and magnon kinetics reveals that while the former can be hydrodynamic, the latter is typically collisionless under most experimental conditions. This implies that, while our zero-temperature hydrodynamic equations are a valid description of spin transport in Bose gases, a hydrodynamic description that treats both mass and spin transport at finite temperatures may not be readily feasible.
\end{abstract}

DOI: 10.1103/PhysRevA.95.053607

\section{INTRODUCTION}

The phenomenon of superfluidity underlies transport properties of numerous systems, including various superconductors [1], liquid helium [2], both bosonic [3] and fermionic [4] ultracold atoms, exciton-polariton condensates [5], topological insulators [6-8], as well as neutron stars [9] and flocks of birds [10]. The possibility to achieve dissipationless propagation of information at room temperature has recently fueled interest in spin superfluidity $[11,12]$ in general and in magnon spintronics [13-15] in particular.

Ferromagnetic spinor Bose-Einstein condensates of atomic vapor stand out among these systems as a rare example where two types of superfluidity can be present simultaneously, and where they are also readily experimentally addressable. Specifically, experimental ultracold atom techniques currently allow controlled excitation and imaging of both the local phase of the condensate, and also of the spin texture. Exciting mass supercurrents (pertaining to inhomogeneity of the local phase of the wave function) in the system is possible by, e.g., stirring the condensate with an "optical spoon" [16]. Signatures of this mass superfluidity have been observed in the collective mode spectrum [17] and lattices of quantized vortices [18]. Furthermore, manipulating and observing the spin texture (or, equivalently, the spin supercurrent [11]) has recently also become possible in this system. In particular, spin-agnostic optical traps [19] have allowed preparation and subsequent imaging of spinor gases, for example, using the Stern-Gerlach method [20] and also directly [21]. Several methods for imprinting spin textures have been developed,

\footnotetext{
*jogundas.armaitis@tfai.vu.lt
}

either relying on varying external magnetic fields [22] or optical transitions [23].

Earlier work [24] has investigated stability of the planar spin spiral ( $X Y$ spiral), which is one of the states that we consider (Fig. 1). Both adiabatic and sudden preparation was carefully considered, and it was demonstrated that this state is stable for sufficiently small spiral wave vectors. However, despite the recent observation of a (quasi)condensate of magnons [25,26], few studies have been devoted to spin superfluidity in ultracold spinor gas [27-30], even though this phenomenon has played a prominent role in liquid helium systems [31]. Furthermore, the interplay of mass and spin superfluidity has not been addressed in the ultracold-atom context to the best of our knowledge.

In this article we examine coherent dynamics of spinor Bose gases, show that these systems may exhibit coexisting superfluidity and spin superfluidity, and study their interplay. We construct a hydrodynamic model that incorporates both types of superfluidity on equal footing. In linear response, they are decoupled. We determine both the collective mode spectrum and demonstrate that spin superfluidity can be experimentally observed by monitoring the in-plane magnetization angle of the atoms. Moreover, we demonstrate that nonlinear effects lead to experimentally stationary states where both supercurrents are counterflowing. Finally, we check the robustness of our findings by comparing the time scales relevant to these coherent processes with the collision time scales describing incoherent dynamics of this system and show that they are well separated.

\section{THEORETICAL FRAMEWORK}

We consider a spinor Bose gas described by the secondquantized Hamiltonian [32-34] $\hat{H}=\hat{H}_{0}+\hat{H}_{Z}+\hat{H}_{I}$, where 


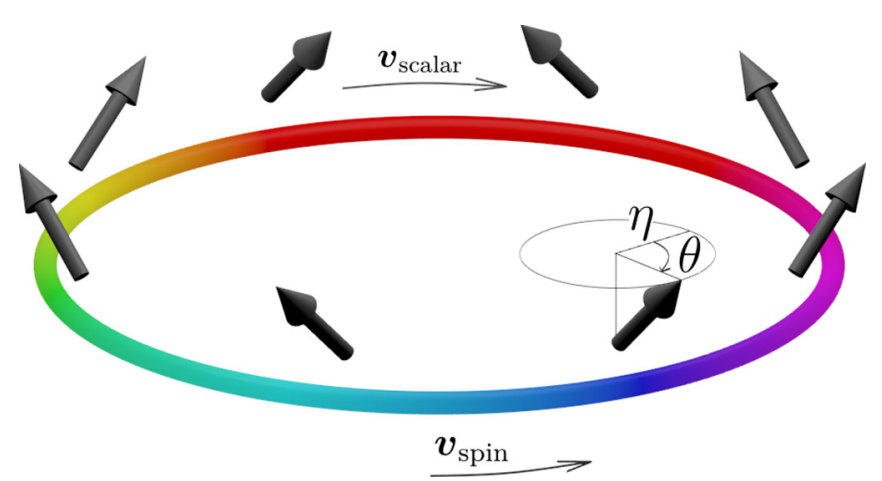

FIG. 1. By counterflowing, a supercurrent and a spin supercurrent create a stationary state with no mass flow in a spinor Bose gas on a ring. In a uniform-density system, the supercurrent $\boldsymbol{v}_{\text {scalar }}$ is due to the global phase texture of the atomic condensate, whereas the spin supercurrent $\boldsymbol{v}_{\text {spin }}$ is due to the in-plane magnetization texture (indicated by arrows which can be recast in terms of a phase $\theta$ ) of the magnon condensate with a measure of the condensate fraction $\eta$. The two textures can be independently engineered in a ferromagnetic spinor Bose gas.

$\hat{H}_{0}=\int d \boldsymbol{x} \hat{\psi}_{\mu}^{\dagger}\left[-\hbar^{2} \nabla^{2} / 2 M+V(\boldsymbol{x})\right] \hat{\psi}_{\mu}$ represents the kinetic energy and the trapping potential $V(\boldsymbol{x})$. The field operators $\hat{\psi}_{\mu}$ correspond to atoms with mass $M$ in the hyperfine state $\mu$. (We use the Einstein summation convention throughout the article.) The linear and quadratic Zeeman effects are described by $\hat{H}_{Z}=-\int d \boldsymbol{x} \hat{\psi}_{\mu}^{\dagger}\left(p\left[\sigma_{z}\right]_{\mu \nu}-\hbar K\left[\sigma_{z}^{2}\right]_{\mu \nu}\right) \hat{\psi}_{\nu}$, where $p(\hbar K)$ is the energy of the linear (quadratic) Zeeman effect [35], and $\sigma_{\mu \nu}$ is a vector of spin matrices. The external magnetic field has been chosen to point in the $z$ direction. We only consider the ferromagnetic state, i.e., the situation where the energy of the linear Zeeman effect is larger than the one of the quadratic Zeeman effect $(p>K)$ [36,37]. Our present treatment is confined to the spin-1 case, which is the lowest-spin system where the quadratic Zeeman term is nontrivial. Extension of our results to higher-spin systems under the same approximations is straightforward. The interactions between particles are described by $\hat{H}_{I}=\int d \boldsymbol{x}\left(g_{0}: \hat{\rho}^{2}:+g_{1}:(\hat{\boldsymbol{n}} \hat{\rho})^{2}:\right) / 2$, where the colons denote normal ordering, $g_{0}$ is the spinindependent interaction strength, $g_{1}$ is the spin-dependent interaction strength, $\hat{\rho}=\hat{\psi}_{\mu}^{\dagger} \hat{\psi}_{\mu}$ is the density operator, and $\hat{\boldsymbol{n}}=\hat{\psi}_{\mu}^{\dagger} \boldsymbol{\sigma}_{\mu \nu} \hat{\psi}_{\nu} / \hat{\rho}$ is the local spin operator. For completeness, we note that the mean-field dynamics derived from this Hamiltonian for the fields $\psi_{\mu}$ corresponding to the aforementioned field operators $\hat{\psi}_{\mu}$ are described by the Gross-Pitaevskii equation $[38,39]$,

$$
\begin{aligned}
i \hbar \partial_{t} \psi_{\mu}= & \left(-\frac{\hbar^{2}}{2 M} \nabla^{2}+V(\boldsymbol{x})\right) \psi_{\mu} \\
& +\left(-p\left[\sigma_{z}\right]_{\mu \nu}+\hbar K\left[\sigma_{z}^{2}\right]_{\mu \nu}+g_{0} \rho\right) \psi_{\mu} \\
& +g_{1} \boldsymbol{n} \cdot \boldsymbol{\sigma}_{\mu \nu} \rho \psi_{\nu},
\end{aligned}
$$

where $\boldsymbol{n}$ is the mean-field magnetization, which corresponds to the operator $\hat{\boldsymbol{n}}$ and is defined as $\boldsymbol{n}=\left\langle\hat{\psi}_{\mu}^{\dagger} \boldsymbol{\sigma}_{\mu \nu} \hat{\psi}_{\nu} / \hat{\rho}\right\rangle$. The mean-field density is defined in a similar manner, $\rho=\langle\hat{\rho}\rangle$. Zero-temperature hydrodynamic equations below are obtained from this Gross-Pitaevskii equation. We note at the outset that the quadratic Zeeman effect will turn out to be crucial in stabilizing the spin superfluidity.

The equations for $\left\langle\hat{\psi}_{\mu}\right\rangle$ governing the mean-field dynamics of this system at zero temperature can be derived from the Hamiltonian $\hat{H}$ and written down in terms of a set of slowly evolving variables. At this point we restrict ourselves to the ferromagnetic state with the saturated local magnetization $\boldsymbol{n}^{2}=1$, which implies that we do not consider the nematic [32-34] or antiferromagnetic [40] evolution. This approximation is well justified only when the gap introduced by the spindependent interaction $\Delta_{\mathrm{FM}}=g_{1} \rho$ is much larger than all the other relevant energy scales, in particular the exchange energy $\hbar J \pi^{2} / L^{2}$, easy-plane anisotropy energy $\hbar K$, and the energy corresponding to the incoherent dynamics (see Sec. IIIE). In that case, the dynamics are confined to the ferromagnetic manifold over the pertinent time scales. Furthermore, we do not consider the trapping potential and the quantum pressure term [3]. Moreover, we omit the linear Zeeman effect, as it can be removed by going to a rotating coordinate system. In order to write down these mean-field equations in a concise manner, we define the usual Eulerian derivative $D_{t}=\partial_{t}+\boldsymbol{v} \cdot \nabla$, where the velocity $\boldsymbol{v}=-i(\hbar / 2 M)\left(\psi_{\mu}^{*} \nabla \psi_{\mu}-\left[\nabla \psi_{\mu}^{*}\right] \psi_{\mu}\right) / \rho$ governs mass transport but has contributions from both the global phase of the wave function and the spin texture. In particular, we find that magnetization dynamics in a ferromagnetic spinor Bose gas is described by a Landau-Lifshitz (LL) equation [28,41]

$$
\begin{aligned}
D_{t} \boldsymbol{n}= & J \boldsymbol{n} \times \nabla^{2} \boldsymbol{n}-K \boldsymbol{n} \times \boldsymbol{e}_{z} n_{z} \\
& +J\left(\boldsymbol{n} \times \nabla_{i} \boldsymbol{n}\right)\left(\nabla_{i} \rho\right) / \rho,
\end{aligned}
$$

where $\rho=\langle\hat{\rho}\rangle$ is the average density (at zero temperature equal to the atomic condensate density) and the exchange constant $J$ comes from the kinetic term in $\hat{H}$ and describes spin stiffness. Neglecting interactions between spin waves, at low temperature the spin stiffness is [42] $J=\hbar / 2 M$. The magnetic anisotropy comes from the quadratic Zeeman effect. It can be generated using a sufficiently strong external magnetic field, in addition to radio frequency and optical means [33]. Three (spin-1) or more hyperfine states are required for the magnetic anisotropy to be available in an atomic system. When $K<0$, it is favorable for the spin to align with the director of the magnetic field. In this case $z$ is known as the easy axis. On the other hand, in the so-called easy-plane situation $K>0$, the configuration with $\boldsymbol{n}$ perpendicular to the $z$ axis is energetically favored. Both of these situations can be achieved in a system of ultracold atoms [33]. In addition to the terms already present in the LL equation, various spin-relaxation terms may be added, such as the transverse spin diffusion [43] and Gilbert damping [44], as well as terms due to magnetic-field inhomogeneities [45] and magnetic dipole-dipole interactions [46]. However, all these terms can be made small in an ultracold-atom system; hence we do not consider them here.

\section{RESULTS}

\section{A. Magnon condensate}

In spinor Bose gases magnetization in the direction of the magnetic field ( $n_{z}$ in this case) is conserved. In this paper, we consider bosons deep in ferromagnetic regime, but with 


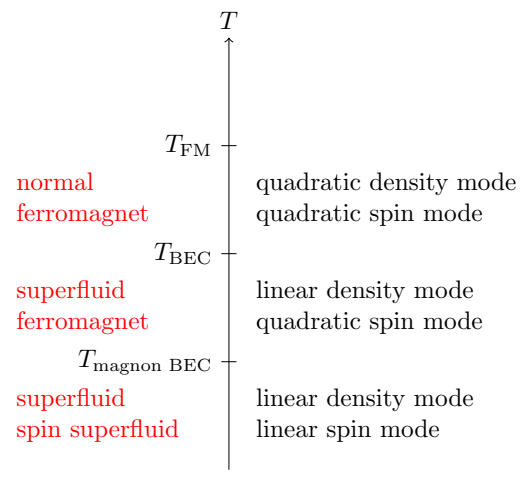

FIG. 2. In a ferromagnetic spinor Bose gas in the presence of a quadratic Zeeman effect, three phase transitions may occur as the temperature is lowered. At high temperature the gas is thermal and has no spin order. Below $T_{\mathrm{FM}}$ the system enters the ferromagnetic phase, characterized by the ordered magnetization (normal ferromagnet). However, both spin and density modes remain quadratic. Below the condensation temperature $T_{\mathrm{BEC}}$ the Bose-Einstein condensate forms, and one of the collective excitations gains a linear Bogoliubov dispersion (superfluid ferromagnet). Finally, below the magnoncondensation temperature $T_{\text {magnonBEC }}$, the dispersion of the spin excitations also become linear (spin superfluid). The presence of two linear collective modes in this low-temperature phase heralds the existence of two types of superfluid currents.

magnetization not saturated in the direction of the magnetic field $\left(n_{z}<|\boldsymbol{n}|\right)$, and thus rotationally invariant around the $z$ axis for sufficiently high temperature. In our case, the phase diagram of the system is altered as compared to the usual treatment [36], where given the same magnetic-field strength $p>K$ only the ferromagnetic phase is allowed. In particular, our setup implies that below a certain temperature $T_{\text {magnonBEC }}$ rotational invariance around the $z$ axis is broken, precession of the magnetization around the magnetic-field direction becomes synchronized, and the system is said to be in the magnon condensate phase. The schematic phase diagram in our case is given in Fig. 2.

Deep in the ferromagnetic regime where the spin excitations are small deviations from the average direction of the magnetization, it is natural to describe the magnetization dynamics in terms of magnons. Going to this description requires performing the Holstein-Primakoff transformation $[47,48]$, which introduces bosonic magnon operators (cf. Sec. IIIE). This gas of magnons can have a thermal component, and also a condensate component. The condensate of magnons [49,50] corresponds to the coherent magnetization precession of the spins in the whole sample. The symmetry that is broken when the magnon condensate forms concerns the in-plane magnetization angle $\theta$, and the absolute value of the order parameter is related to the out-of-plane magnetization component $n_{z}$; cf. Eq. (4) and below.

Since we concentrate on the superfluidity in this system, we do not describe the thermal magnons, and we also do not investigate the complicated magnon condensation process [26]. However, we remark for completeness that in a ferromagnetic spinor Bose gas in the presence of a quadratic Zeeman effect with $n_{z}$ conserved, three phase transitions may occur as the temperature is lowered (see Fig. 2). The well-known Bose-Einstein condensation in the mean-field theory occurs at [3]

$$
T_{\mathrm{BEC}}=\frac{2 \pi \hbar^{2}}{k_{B} M}\left(\frac{\rho}{\zeta_{3 / 2}}\right)^{2 / 3},
$$

where $k_{B}$ is the Boltzmann constant and $\zeta_{3 / 2} \simeq 2.612$. The order parameter of the BEC phase is proportional to the expectation value of the atom annihilation operator.

At a temperature $T_{\mathrm{FM}}$, which at the mean-field level is higher than but similar to $T_{\mathrm{BEC}}[51,52]$, the system becomes ferromagnetically ordered. The global magnetization is the order parameter defining the ferromagnetic state, and it becomes nonzero as the system enters the ferromagnetic state. However, in the system we consider, $n_{z}$ is conserved, and thus the global magnetization in the $z$ direction cannot be a function of temperature. Therefore, the ferromagnetic transition manifests itself in a subtler than usual manner. For example, spatial regions emerge with either a nonzero in-plane magnetization [20], or with different values of $n_{z}$ [53]; see Ref. [33] for a more thorough discussion. Most importantly for our purposes, deep in the ferromagnetic state the local magnetization is fully saturated $(|\boldsymbol{n}|=1)$ throughout the system irrespective of conservation of $n_{z}$.

Finally, if $p<\hbar K$, the system enters the magnon condensate phase at $T_{\text {magnonBEC }} \simeq T_{\mathrm{BEC}}(1-p / \hbar K)^{2 / 3}$ [54]. The magnon condensate is equivalent to the broken axisymmetry phase in the full thermodynamic phase diagram of the system [36,37], with the crucial difference that whereas the total magnetization is still saturated $(|\boldsymbol{n}|=1)$ in the magnon condensate phase, in the broken axisymmetry phase it is not $(|\boldsymbol{n}|<1)$.

In general, during the process of condensate formation, a certain symmetry is broken, leading to a nonzero order parameter. In the case of Bose-Einstein condensation of atoms, the symmetry concerns the phase of the wave function, and the order parameter is the average of the atom-annihilation operator. In the condensate of magnons [49,50], the symmetry concerns the in-plane magnetization angle $\theta$, and the order parameter is related to the out-of-plane magnetization component $n_{z}$. It is important to point out that the LandauLifshitz equation explicitly preserves the local magnetization $|\boldsymbol{n}|$ in stark contrast to the dynamics of a BEC of atoms, where no such order-parameter conservation law exists in the grand-canonical description [55]. Therefore, we expect the magnon condensate formation to differ from the process of Bose-Einstein condensation of atoms.

In order to avoid various complications associated to the magnon condensate formation, we propose to start from a sample that is homogeneously magnetized in the $z$ direction, and then coherently tilt the magnetization (e.g., by an RF pulse) towards the $x-y$ plane. This populates the pure-magnoncondensate state directly by preparing the magnetization of the system in the following configuration:

$$
\boldsymbol{n}=\left(\eta \cos \theta, \eta \sin \theta, \sqrt{1-\eta^{2}}\right),
$$

where $\eta=\sqrt{2 \tilde{\eta} /\left(1+\tilde{\eta}^{2}\right)}$ is a measure of the local magnoncondensate fraction $\tilde{\eta}$ [56] and $\theta$ is the local in-plane angle. That this magnetization configuration indeed corresponds to a magnon condensate is understood by noting that $n_{x} \sim \operatorname{Re}\langle\hat{b}\rangle$ 
and $n_{y} \sim \operatorname{Im}\langle\hat{b}\rangle$, where $\hat{b}$ is the Holstein-Primakoff operator that annihilates a magnon.

Using Eq. (4) together with the Landau-Lifshitz equation Eq. (2), and using the mean-field equations for the total density, we obtain a set of hydrodynamic equations, governing the evolution of the magnon condensate and the scalar condensate at zero temperature:

$$
\begin{gathered}
D_{t} \rho / \rho=-\nabla \cdot \boldsymbol{v}, \quad M D_{t} \boldsymbol{v}=-\nabla \mu, \\
D_{t} n_{z}+\boldsymbol{v}_{\text {spin }} \cdot \nabla \rho / \rho=-\nabla \cdot \boldsymbol{v}_{\text {spin }}, \quad \hbar D_{t} \theta=-\mu_{\text {spin }},
\end{gathered}
$$

where $\mu=\left(g_{0}+g_{1}\right) \rho+\hbar^{2}\left(\nabla n_{\mu}\right)^{2} / 4 M$ and $\mu_{\text {spin }} / \hbar J=$ $n_{z}(\nabla \theta)^{2}-\left(\nabla^{2} \eta\right) / n_{z} \eta-(\nabla \eta)^{2} / n_{z}^{3}-K n_{z} / J$ are the chemical potentials. Their gradients drive the mass and spin supercurrents. Note that the quantum pressure term [3] has been neglected from the scalar condensate equations, as we will only be interested in states of uniform particle density from hereon. Therefore, these hydrodynamic equations only describe density dynamics on length scales longer than the condensate healing length. On the other hand, similar terms have been kept for now in $\mu_{\text {spin }}$ since we consider an inhomogeneous magnon condensate in Sec. III D. Furthermore, even though similar equations have been derived in previous work [32-34,40,41], here we describe spin and mass superfluidity in a single framework. Hence these hydrodynamic equations constitute the central result of our article.

The total velocity can be separated into two parts [32] that can be addressed separately $[16,22,23]$. First, we have the conventional superfluid velocity $\boldsymbol{v}_{\text {scalar }}=(\hbar / M) \nabla \phi$ due to the phase $\phi$ texture of the atomic condensate wave function. However, there also is the spin superfluid velocity $\boldsymbol{v}_{\text {spin }}=$ $-J \eta^{2} \nabla \theta$ due to the phase $\theta$ texture of the magnon condensate. Thus the full velocity in this ferromagnetic spinor Bose condensate is

$$
\boldsymbol{v}=\boldsymbol{v}_{\text {scalar }}+2 n_{z} \boldsymbol{v}_{\text {spin }} / \eta^{2} .
$$

This velocity $v$ influences the magnetization dynamics through the advection term in the Eulerian derivative since the magnetic moments (individual atoms) are mobile in a cold-atom system, similar to, e.g., so-called ferromagnetic superconductors [57]. This is in contrast to most solid-state ferromagnets, where the magnetic moments are localized.

\section{B. Linear regime and collective modes}

In the linear regime, the two superfluids are decoupled as the Eulerian derivatives become ordinary derivatives. The elementary excitations on top of the homogeneous magnon condensate $(\eta, \theta=$ const $)$ have a dispersion

$$
\omega^{2}=K J \eta^{2} k^{2}+J^{2} k^{4},
$$

which follows from the equations of motion above, and is linear in the long-wavelength limit [58]. Moreover, the dispersion of the density excitations follow the Bogoliubov dispersion [33],

$$
\omega^{2}=(\hbar / 2 M) k^{2}\left[(\hbar / 2 M) k^{2}+2\left(g_{0}+g_{1}\right) \rho / \hbar\right],
$$

the derivation of which requires keeping the quantum pressure terms (see Ref. [32] for an explicit calculation). At long wavelengths the dispersion is linear in both cases, with the speed of sound equal to $c_{\mathrm{spin}}=\sqrt{K J \eta^{2}}$ for the spin excitations and $c_{\text {scalar }}=\sqrt{\left(g_{0}+g_{1}\right) \rho / M}$ for the density excitations, signaling that we are dealing with a superfluid, as using the Landau argument [59] one can show that excitations traveling with velocities slower than the lower of these two speeds of sound are not damped. Note that in general spinor gases are not necessarily spin superfluid in the ferromagnetic regime. In particular, the spin currents discussed in Ref. [60] in the absence of quadratic Zeeman effect are not spin supercurrents, as their critical velocity vanishes. From this point on we only consider the critical spin superfluid velocity and drop its subscript: $c \equiv c_{\text {spin }}$. Note that this homogeneous state is stable and displays spin superfluidity in the easy-plane situation $K>0$ only. Furthermore, note that tilting the magnetization, i.e., considering the magnon condensate in the ferromagnetic phase, results in a spectrum that is distinct from both the usual collective modes in the ferromagnetic phase as well as the broken axisymmetry phase (cf. the appendixes of Ref. [36]).

In order to verify that the system is indeed a spin superfluid and to show that the conventional superfluidity and the spin superfluidity stand on an equal footing, we propose two experiments which should be realizable with current experimental techniques. To keep the description as simple as possible, we work in the one-dimensional limit, where the spatial confinement is strong in two dimensions and more gentle in the remaining spatial dimension. Furthermore, in order to avoid complications due to trap averaging, we consider a box trap [61]. A boxlike potential of a form similar to the one depicted in Fig. 1 has already been experimentally produced and persistent currents of a scalar superfluid have been observed in such a setup in Ref. [62].

\section{Far-from-equilibrium spin superfluidity signature in the nonlinear regime}

One of the hallmarks of superfluidity is an unobstructed flow of current. In particular, in a spin superfluid, a spin current can flow with no dissipation as opposed to a system with diffusive spin transport [63], where the spin current decays after traversing some finite length, which depends on the diffusion length and on the time scales of various spin relaxation mechanisms [11]. In this section we describe a stationary state (Fig. 1) where the supercurrent and the spin supercurrent flow in opposite directions, resulting in no mass transport. However, since the spin current flows through the whole sample, it thus illustrates dissipationless spin transport [11].

By considering linear gradients in the atomic condensate phase $\phi$ and in the magnon condensate phase $\theta$ with a constant magnon condensate fraction $\eta$, from Eqs. (6) we find the stationary-state condition

$$
\phi^{\prime}=\frac{n_{z}}{2}\left(\theta^{\prime}+\frac{K}{J \theta^{\prime}}\right),
$$

where the primes indicate spatial derivatives. In order to show that this condition can be satisfied for realistic experimental parameters, we consider a concrete example of a ring of length $L$ filled with a cloud of spin-1 atoms (e.g., the $F=1$ hyperfine manifold of the strongly ferromagnetic ${ }^{7} \mathrm{Li}$ ) prepared 
in the magnon-condensate state described by Eq. (4). In that case, a state with $n_{z}=1 / 2$ where the atomic condensate phase winds once $\left(\phi^{\prime}=2 \pi / L\right)$, while the magnon condensate phase has two windings $\left(\theta^{\prime}=2 \times 2 \pi / L\right)$, is stationary for $K L^{2} / \pi^{2} J=16$. For a ring of length $L=50 \mu \mathrm{m}$, we have $J \pi^{2} / L^{2} \simeq 20 \mathrm{~Hz}$, which requires a moderate easy-plane anisotropy of circa $300 \mathrm{~Hz}$. As explained in Sec. II, in order for our theory to be applicable, the gap $\Delta_{\mathrm{FM}}=g_{1} \rho$ has be larger than any other energy scale relevant to the dynamics. This requirement can be satisfied for a strongly ferromagnetic gas of a sufficiently high density. In the case of the stable $F=1$ manifold of the ${ }^{7} \mathrm{Li}$ atom [33], a relatively high but experimentally feasible density of $10^{20} \mathrm{~m}^{-3}$ leads to a gap of $\Delta_{\mathrm{FM}} / \hbar=3.5 \mathrm{kHz}$, which is well above any other energy scales relevant to the dynamics considered here. Moreover, this static state implies no mass transport as $\boldsymbol{v}_{\text {scalar }}$ exactly cancels $\boldsymbol{v}_{\text {spin }}$, and thus $\boldsymbol{v}=0$. Furthermore, this stationary state is also stable according to the relevant Landau criteria [59]. When it comes to the spin supercurrent, $\left|\boldsymbol{v}_{\text {spin }}\right| / c_{\text {spin }}=1 / 2<1$. Concerning density excitations, the criterion is satisfied due to the large gap $\Delta_{\mathrm{FM}}$ for the densities in question, namely,

$$
\left|\boldsymbol{v}_{\text {scalar }}\right| / c_{\text {scalar }}=\sqrt{8 \frac{J \pi^{2} / L^{2}}{\left(g_{0}+g_{1}\right) \rho / \hbar}} \ll 1 .
$$

Note that without easy-plane anisotropy, and therefore without spin superfluidity, such steady states cannot be obtained.

In particular, we would like to emphasize the difference between the state described in this subsection and the fractional vortices in polar spinor condensates. When it comes to the polar (nonmagnetic) phase, the dispersion of spin amplitude or the nematic angle is indeed linear at long wavelengths, signaling a nonzero critical (Landau) velocity for these excitations [32]. However, the dynamics of this system is governed by the nematic director, in sharp contrast to conventional magnetism governed by the magnetization vector dynamics. More formally, the order-parameter space in the polar phase is $U(1) \times S^{2} / \mathbb{Z}_{2}$. This structure comes from the fact that a $\pi$ rotation of the scalar phase in combination with inverting the nematic director leaves a polar state unchanged. Indeed, this symmetry in particular allows fractional vortices to exist in the polar state. Therefore, even though the current defined in terms of the gradients of the nematic director is sometimes called the spin supercurrent [60], it is in our opinion to be distinguished from the usual case, i.e., from the spin current defined in terms of a gradient of magnetization direction, as is conventional in other systems, such as solid-state magnets. Finally, in the ferromagnetic phase of spin-1 bosons that is considered in this article, the order parameter is indeed the magnetization vector, and its space is the usual $\mathrm{SO}(3)$. In that case the mass current has a spin-texture contribution [cf. Eq. (7)], which allows us to find the stationary states described in our work. In the special case of easy-plane anisotropy the spin current carried by the texture is a supercurrent. No such spin-texture term exists in the polar phase [60], and thus the physical mechanism behind the half-quantum vortices is in our opinion remarkably different from the stationary states that we propose.
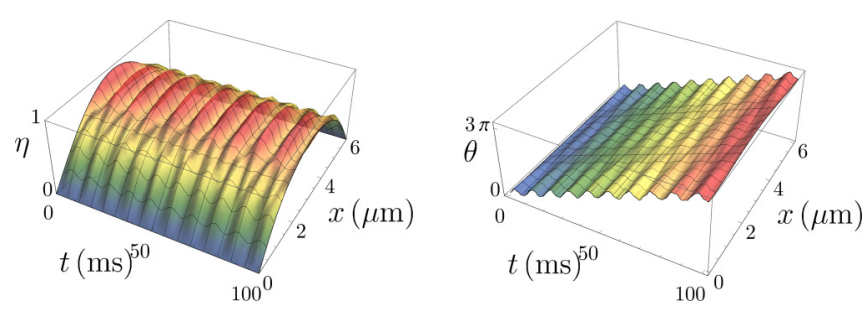

FIG. 3. Evolution of a sine-shaped magnon condensate packet for realistic experimental parameters: an $L=20-\mu \mathrm{m}$-long cloud of strongly ferromagnetic ${ }^{7} \mathrm{Li}$ atoms, with the resulting exchange $J \pi^{2} / L^{2} \simeq 100 \mathrm{~Hz}$, easy-plane anisotropy of $K=300 \mathrm{~Hz}$, and a peak initial condensate density of $\eta_{0}=0.95$ according to Eqs. (6) with no approximations. The density of the condensate $\eta$ (left) is almost unaffected by the dynamics, while the in-plane angle $\theta$ (right) evolves almost uniformly (see Sec. IIID for more details). The in-plane magnetization completes a full rotation in approximately $60 \mathrm{~ms}$. Note that the total magnetization in the $z$ direction is conserved during the evolution.

\section{Close-to-equilibrium spin superfluidity signature}

It is also possible to observe a signature of spin superfluidity by measuring the time evolution of the in-plane magnetization angle $\theta$ in the simple "bar" geometry (as opposed to the ring discussed above). To that end, consider a magnon condensate with a constant atomic condensate phase $\phi$ and a constant in-plane angle $\theta$, in addition to a smoothly varying magnon condensate fraction bump $\eta=\eta_{0} \sin (\pi x / L)$ at the initial time $t=0$, where $L$ is the length of the atomic cloud and $x$ is the spatial coordinate; $\mathrm{cf}$. Fig. 3 . In this case, up to the lowest order in the gradient expansion, the time evolution preserves the magnon-condensate-density profile such that $\partial_{t} \eta=0$, while the in-plane angle rotates in time with no spatial profile developing,

$$
\partial_{t} \theta=K\left\langle n_{z}\right\rangle-J \pi^{2} /\left\langle n_{z}\right\rangle L^{2},
$$

where $\left\langle n_{z}\right\rangle=2 \mathcal{E}\left[\eta_{0}^{2}\right] / \pi$ is the $z$ component of magnetization averaged over the length of the cloud and $\mathcal{E}$ is the complete elliptic integral.

We now connect this inhomogeneous setup with the magnon dispersion of the homogeneous system in Eq. (8). On the one hand, local-density approximation [64] is applicable only when the relevant quantity ( $\eta$ in this case) varies slowly enough with respect to the appropriate coherence length (also known as the healing length) $\xi$ [3]. On the other hand, a system with a linear dispersion can only be considered superfluid if its evolution actually probes the linear part of the dispersion. Here the healing length

$$
\xi=\sqrt{\frac{J}{K}} \frac{1}{\eta}
$$

is obtained by comparing the two terms in Eq. (8). The relevant length scale to compare with $\xi$ is in this case the length of the atomic cloud $L$. In the center of the cloud, where $\eta \simeq 1$, using the parameters in the caption of Fig. 3 , we have $\xi / L \simeq 0.2 \ll 1$. Therefore, we conclude that the local-density approximation is applicable in the central part of the cloud. We now check if the linear part of the dispersion is actually probed. Since the longest possible wavelength is equal to the system size, $L$, we 
plug the wave vector $k=\pi / L$ into the dispersion relation in Eq. (8) and obtain $(\pi / L)^{-2} \omega^{2}=K J \eta^{2}+J^{2}(\pi / L)^{2}$. Thus, to see if the linear part of the dispersion is relevant, we have to compute the ratio between two sound velocities, namely, $c^{2}=$ $K J \eta^{2}$ and $c_{0}^{2}=J^{2}(\pi / L)^{2}$. Furthermore, since $c^{2}$ is position dependent, we have to average it: $\left\langle c^{2}\right\rangle=K J \eta_{0}^{2} / 2$. It can be concluded that spin superfluidity becomes pronounced when

$$
\frac{\left\langle c^{2}\right\rangle}{c_{0}^{2}}=\frac{K J\left\langle\eta^{2}\right\rangle}{J^{2}(\pi / L)^{2}}=\frac{K \eta_{0}^{2}}{2 J(\pi / L)^{2}}>1 .
$$

This criterion can be readily evaluated experimentally by observing the evolution of the in-plane angle. In particular, the evolution stops $\left(\partial_{t} \theta=0\right)$ when $K L^{2}\left\langle n_{z}\right\rangle^{2} / J \pi^{2}=1$. Therefore, prevalence of spin superfluidity in the sample for large condensate fractions $\left(\eta_{0}>0.95\right)$ is proven by measuring that $\partial_{t} \theta>0$, since $\mathcal{E}\left[\eta_{0}^{2}\right]$ is a monotonically decreasing function, and since $\left\langle n_{z}\right\rangle^{2}\left(\eta_{0} \simeq 0.95\right) \simeq 1 / 2$.

Hence, for a large condensate fraction $\eta$, observing the evolution of $\theta$ is sufficient to distinguish between the situation where the critical velocity $c$ vanishes and where it is substantial. Namely, if $\partial_{t} \theta<0$, the system is dominated by quadratic excitations and has a negligible critical velocity, whereas if $\partial_{t} \theta>0$, the system displays spin superfluidity. In order to illustrate that our conclusion holds for the full solution for realistic experimental parameters, we plot the numerical solution of Eqs. (6) with the boundary conditions $\eta(0)=\eta(L)=0$ and $\theta^{\prime}(0)=\theta^{\prime}(L)=0$ and the aforementioned initial conditions in Fig. 3.

\section{E. Incoherent dynamics}

Up to this point we have only considered coherent magnetization dynamics. However, due to nonzero temperatures and interparticle collisions present in real ultracold-atom systems, it is important to investigate incoherent (kinetic) processes as well. In particular, it is interesting to study the time scales of the incoherent (kinetic) magnon dynamics and compare them to the time scales of the coherent evolution. We compare these two time scales by evaluating the dominant incoherent time scale set by the four-magnon interaction [58,65-67] kinetic integral, which we obtain by using the HolsteinPrimakoff transformation [47] in order to describe the spin degrees of freedom in this ultracold-atom system in terms of magnons. In particular, we compute that the coherent dynamics discussed previously is an order of magnitude faster (tens of milliseconds) than the incoherent processes (hundreds of milliseconds) for an experimentally accessible system (see Refs. [23,45]). This implies that, while our hydrodynamic description is valid at zero temperature, at finite temperatures the thermal magnons do not equilibrate on the time scales set by the coherent dynamics, which in turn means a hydrodynamic description of the spin dynamics cannot be readily obtained at finite temperature.

In order to compute the four-magnon scattering time, we consider the exchange term in the spinor Bose gas Hamiltonian, namely, $J: \hat{\mathbf{\Omega}} \cdot \nabla^{2} \hat{\boldsymbol{\Omega}}:$, where $\hat{\boldsymbol{\Omega}}$ is the full magnetization operator. It can be divided into the magnetization density $\rho_{s}$, which we assume to be constant in the deep ferromagnetic regime, and the direction of magnetization operator $\hat{\boldsymbol{n}}$ in the following way: $\hat{\boldsymbol{\Omega}}=\rho_{s} \hat{\boldsymbol{n}}$. Hence the magnetic excitations only concern the direction of magnetization $\boldsymbol{n}$ in this regime. Since we wish to merely compare the order of magnitude of the coherent and incoherent dynamics, we do not consider the quadratic Zeeman effect in this calculation. As discussed below, making reliable quantitative predictions requires not only including the quadratic Zeeman effect, but also taking into account magnetic-field inhomogeneity. The Holstein-Primakoff transformation $[47,48]$ introduces bosonic magnon operators $\hat{b}(\boldsymbol{k})$, which substitute for the magnetization direction operators. We subsequently perform a semiclassical expansion of these spin fluctuations around the average direction of the magnetization, and retain only two first terms in this large-magnetization expansion.

The lowest-order term yields the kinetic energy for the magnons,

$$
\mathcal{H}_{2}=\int \frac{d \boldsymbol{k}}{(2 \pi)^{3}} E_{k} \hat{b}^{\dagger}(\boldsymbol{k}) \hat{b}(\boldsymbol{k}),
$$

where $E_{k}=\hbar J \boldsymbol{k}^{2}$ is the magnon dispersion [68,69], whereas the subleading term describes the four-magnon interaction,

$$
\begin{aligned}
\mathcal{H}_{4}= & \int \frac{d \boldsymbol{k}}{(2 \pi)^{3}} \frac{d \boldsymbol{k}_{2}}{(2 \pi)^{3}} \frac{d \boldsymbol{k}_{3}}{(2 \pi)^{3}} \frac{d \boldsymbol{k}_{4}}{(2 \pi)^{3}} \delta^{(3)}\left(\boldsymbol{k}+\boldsymbol{k}_{2}-\boldsymbol{k}_{3}-\boldsymbol{k}_{4}\right) \\
& \times g \hat{b}^{\dagger}(\boldsymbol{k}) \hat{b}^{\dagger}\left(\boldsymbol{k}_{2}\right) \hat{b}\left(\boldsymbol{k}_{3}\right) \hat{b}\left(\boldsymbol{k}_{4}\right)
\end{aligned}
$$

where

$$
g=\frac{\hbar J}{4 \rho_{s}}\left(\boldsymbol{k} \cdot \boldsymbol{k}_{2}+\boldsymbol{k}_{3} \cdot \boldsymbol{k}_{4}\right)
$$

is the four-magnon coupling constant. Note that this coupling constant $g$ does not explicitly depend on the scattering properties of the particular atom. We also note that including the easy-plane anisotropy resulting from the quadratic Zeeman shift leads to a constant (momentum independent) contribution to the scattering amplitude that we ignore here. From this interaction term, using, e.g., the Fermi golden rule, we construct a collision integral. The characteristic time scale is thus given by

$$
\begin{aligned}
\frac{1}{\tau_{k}}= & \frac{2 \pi}{\hbar} \int \frac{d \boldsymbol{k}_{2}}{(2 \pi)^{3}} \int \frac{d \boldsymbol{k}_{3}}{(2 \pi)^{3}} \int \frac{d \boldsymbol{k}_{4}}{(2 \pi)^{3}} g^{2} \mathcal{F} \\
& \times \delta\left(E_{\boldsymbol{k}}+E_{\boldsymbol{k}_{2}}-E_{\boldsymbol{k}_{3}}-E_{\boldsymbol{k}_{4}}\right)(2 \pi)^{3} \\
& \times \delta^{(3)}\left(\boldsymbol{k}+\boldsymbol{k}_{2}-\boldsymbol{k}_{3}-\boldsymbol{k}_{4}\right),
\end{aligned}
$$

where

$$
\mathcal{F}=f_{2}\left(1+f_{3}\right)\left(1+f_{4}\right)-\left(1+f_{2}\right) f_{3} f_{4}
$$

is a combination of Bose-Einstein distributions $f_{i}=$ $1 /\left[\exp \left(\beta E_{\boldsymbol{k}_{i}}\right)-1\right]$, and $\beta=1 / k_{B} T$ is the inverse thermal energy.

This time scale can be written in the dimensionless form,

$$
\frac{\hbar \beta_{c}}{\tau_{k \Lambda}}=\left(\frac{T}{T_{c}}\right)^{4} I(k \Lambda),
$$

where $\Lambda$ is the thermal magnon de Broglie wavelength, $k_{B} T_{c} \simeq 4 \pi \hbar J\left(\rho_{m} / 2.6\right)^{2 / 3}$ is the condensation temperature of a noninteracting homogeneous magnon gas with density $\rho_{m}$, and $I(k \Lambda)$ is a dimensionless function, which we evaluate numerically (see Fig. 4). 


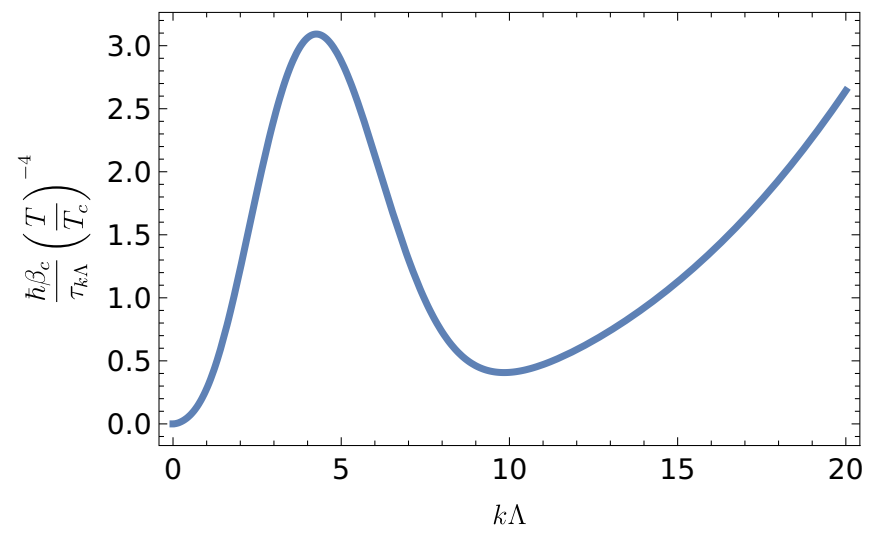

FIG. 4. Typical incoherent dynamics time scale evaluated from the four-magnon interaction term.

Note that for small $k \Lambda$, the kinetic frequency $\tau_{k \Lambda}^{-1}$ approaches zero; as $k \Lambda$ increases, $\tau_{k \Lambda}^{-1}$ has a local maximum and a local minimum, and only then does it approach the classical limit. These features can be understood in terms of competition between the Bose enhancement and the four-magnon coupling constant, as the former favors low $k \Lambda$ scattering, while the latter suppresses it. At low momenta, fitting $I$ yields a function $(k \Lambda)^{2} \ln k \Lambda$, similar to the one reported previously in a field-theoretical calculation $[58,67]$.

Before comparing the result of our kinetic theory with experimental findings, we note that our result is the lower bound on the relaxation frequency, since we have only included a single relaxation process. It is likely that due to other processes (e.g., related to magnetic-field gradients), the relaxation is faster. This statement is consistent with the fact that for a realistic system our result is $1 / \tau \simeq 0.2 \mathrm{~Hz}$, i.e., an order of magnitude lower than the recently reported experimental rate [23]. The comparison is performed using the following data. From Fig. 1(b) in the paper on coherent magnon optics (Ref. [23]) we read off that $1 / \tau \simeq 4 \mathrm{~Hz}$ for $k=2 \pi /(15.4 \mu \mathrm{m})$. No temperature is given in the paper, but assuming that $T=T_{c} / 10$, and taking $T_{c} \simeq 1 \mu \mathrm{K}$ from another paper of the same group (Ref. [45]), we have $k \Lambda \simeq 1 / 4$ and $\hbar \beta_{c} \simeq 10^{-5} \mathrm{~s}$. These assumptions yield $1 / \tau \simeq 0.2 \mathrm{~Hz}$, which is one magnitude lower than the experimental result [23]. Note that it is possible to improve our calculation by performing an accurate trap average, distinguishing between magnon and gas condensation temperatures and populations, etc.

As discussed above, while our hydrodynamic description is valid at zero temperature, at finite temperatures the thermal magnons do not equilibrate on the time scales set by the coherent dynamics, which in turn means a hydrodynamic description of the spin dynamics cannot be readily obtained at finite temperature. This stands in contrast to the situation regarding the scalar degrees of freedom [70] in this system. The difference is mainly due to the different coupling constants, as the four-magnon coupling constant depends on the density and momenta of the magnons, whereas for the scalar degrees of freedom the coupling constant only depends on the $s$-wave scattering length $a$ of the atom [71]. A typical collision time of atoms in the classical approximation [72] is $\tau_{\mathrm{cl}}=1 / n \sigma v_{\text {rel }}$, where $n$ is the density, $\sigma=8 \pi a^{2}$ is the collision cross section, and $v_{\text {rel }}$ is the relative thermal velocity. For a homogeneous ${ }^{7} \mathrm{Li}$ gas with a density of $10^{20} \mathrm{~m}^{-3}$ considered in Secs. III C and III D, the condensation temperature is $5 \mu \mathrm{K}$. At one-tenth of this temperature, the collision time of atoms is $2 \mathrm{~ms}$ [73]. Therefore, at a nonzero temperature such a system is in the hydrodynamic regime regarding the scalar degrees of freedom, which is enforced by rapid collisions. On the other hand, given the same conditions the magnon dynamics is in the collisionless regime, allowing for far-from-equilibrium states to persist for long periods of time. We reiterate that this time-scale hierarchy is well defined, as different time scales are separated by at least one order of magnitude. Finally, the above-described situation concerning magnons in a spinor gas is also different from the binary-mixture situation. There, longitudinal spin kinetics is comparable to the incoherent dynamics of the scalar fields, and hence longitudinal spin currents relax in milliseconds as well $[74,75]$.

\section{F. Lower and upper spin currents}

In a general spin superfluid, in addition to an easy-plane anisotropy $K$, an $n_{x}$-fold in-plane anisotropy $K_{x}$ can play an important role $[11,13]$. This in-plane anisotropy favors the magnetization to point along one of the $n_{x}$ axes in the easy plane. The interplay between the exchange energy and each of these two different anisotropies defines the upper critical spin current $j_{c \text {,up }}$ and the lower critical current $j_{c \text {,low }}$, respectively.

In particular, when the in-plane spin rotation $|\nabla \theta|$ is so large that the corresponding spin current $j$ exceeds $j_{c \text {,up }}$, this spin texture can relax by escaping the easy plane, i.e., acquiring a magnetization component along the hard axis. The upper critical spin current can thus be estimated by equating the exchange energy and the energy of the easy-plane anisotropy:

$$
J|\nabla \theta|_{c, \text { up }}^{2}=\hbar K \text {. }
$$

On the other hand, if the in-plane spin rotation $|\nabla \theta|$ is sufficiently small $\left(j<j_{c}\right.$,low $)$, a single spin domain wall arises, thus preventing superfluid spin flow over macroscopic distances. Hence the lower critical spin current can be derived by equating the exchange energy and the energy corresponding to the in-plane anisotropy:

$$
J|\nabla \theta|_{c, \text { low }}^{2}=n_{x} \hbar K_{x}
$$

Therefore, for a large range of spin currents to be allowed, it is required that $j_{c \text {, low }} \ll j_{c \text {,up }}$, or, equivalently, the inplane anisotropy has to be much weaker than the easy-plane anisotropy: $K_{x} \ll K$.

When it comes to cold-atom systems, the easy-plane anisotropy $K$ is well-controlled and can be made large compared to the exchange energy, as described in the main text. We expect that the in-plane anisotropy, however, will be chiefly caused by stray magnetic fields. It thus will presumably be small as compared to the easy-plane anisotropy, and already controlled for in the experiment for other reasons. We note further that magnetic dipole-dipole interactions can also give rise to a lower critical spin current. However, for single atoms these magnetic dipole-dipole interactions are usually weak, and can be minimized by choosing a suitable atomic species; see Ref. [76] for a review. For the two states proposed in 
the article, the ratios between the exchange energy and the easy-plane anisotropy are 0.06 (see Sec. III C) and 1/3 (see the caption of Fig. 3). We are hence confident that both proposed states correspond to spin currents, which belong to the region between the lower and the upper critical spin currents.

\section{SUMMARY AND FUTURE WORK}

In summary, we have examined the zero-temperature coherent dynamics of spinor Bose gases with a quadratic Zeeman effect and shown that this system exhibits coexisting spin and mass superfluidity. We have described two experimentally accessible states illustrating the interplay between superfluidity and spin superfluidity. By evaluating a four-magnon collision integral and comparing the relevant time scales, we have concluded that at nonzero temperatures the magnon gas is in the collisionless regime in stark contrast to the situation concerning the scalar degrees of freedom.

Experimental access to the states described in this work is constrained by the condition that the spin-dependent interaction has to be much larger than all the other relevant energy scales. At present, it seems that this constraint can be most readily satisfied using strongly ferromagnetic atomic species, such as ${ }^{7} \mathrm{Li}$. However, other ways of addressing this restriction, such as employing Feshbach resonances [77] to enhance interactions, or investigating systems with high particle density, e.g., the ${ }^{3} \mathrm{He}$ liquid [78] or solid-state superconductors with spin order [79], might be possible.
In future work, we plan to extend our description by including the experimentally relevant magnetic-field inhomogeneities into our LL equation, which will allow us to make a direct connection to the recent experimental results on magnon condensation [26,45]. Another interesting direction is to consider nonzero temperature dynamics of scalar and spin degrees of freedom, which is particularly intriguing in this system due to a clear hierarchy of time scales involved. Finally, we point out that coupling between magnon condensate and thermal cloud is facilitated by the quadratic Zeeman term [54], which could make the experimental control over the build-up of magnon coherence and condensate growth possible. Moreover, this anisotropy will also affect the magnon kinetics.

\section{ACKNOWLEDGMENTS}

It is our pleasure to thank Henk Stoof and Gediminas Juzeliūnas for stimulating discussions. J.A. has received funding from the European Union's Horizon 2020 research and innovation programme under the Marie SkłodowskaCurie Grant Agreement No. 706839 (SPINSOCS). R.A.D. is supported by the Stichting voor Fundamenteel Onderzoek der Materie (FOM) and is part of the D-ITP consortium, a program of the Netherlands Organisation for Scientific Research (NWO) that is funded by the Dutch Ministry of Education, Culture and Science (OCW).
[1] Novel Superfluids, edited by K.-H. Bennemann and J. B. Ketterson, International Series of Monographs on Physics (Oxford University Press, Oxford, 2013).

[2] I. M. Khalatnikov, An Introduction to the Theory of Superfluidity (Westview Press, Boulder, 2000).

[3] C. J. Pethick and H. Smith, Bose-Einstein Condensation in Dilute Gases, 2nd ed. (Cambridge University Press, Cambridge, UK, 2008).

[4] L. A. Sidorenkov, M. K. Tey, R. Grimm, Y.-H. Hou, L. Pitaevskii, and S. Stringari, Second sound and the superfluid fraction in a Fermi gas with resonant interactions, Nature (London) 498, 78 (2013).

[5] H. Deng, H. Haug, and Y. Yamamoto, Exciton-polariton BoseEinstein condensation, Rev. Mod. Phys. 82, 1489 (2010).

[6] X.-L. Qi, T. L. Hughes, S. Raghu, and S.-C. Zhang, TimeReversal-Invariant Topological Superconductors and Superfluids in Two and Three Dimensions, Phys. Rev. Lett. 102, 187001 (2009).

[7] D. Tilahun, B. Lee, E. M. Hankiewicz, and A. H. MacDonald, Quantum Hall Superfluids in Topological Insulator Thin Films, Phys. Rev. Lett. 107, 246401 (2011).

[8] S. Peotta and P. Törmä, Superfluidity in topologically nontrivial flat bands, Nat. Commun. 6, 8944 (2015).

[9] D. J. Dean and M. Hjorth-Jensen, Pairing in nuclear systems: From neutron stars to finite nuclei, Rev. Mod. Phys. 75, 607 (2003).

[10] A. Attanasi, A. Cavagna, L. Del Castello, I. Giardina, T. S. Grigera, A. Jelić, S. Melillo, L. Parisi, O. Pohl, E. Shen, and M. Viale, Information transfer and behavioural inertia in starling flocks, Nat. Phys. 10, 691 (2014).
[11] E. B. Sonin, Spin currents and spin superfluidity, Adv. Phys. 59, 181 (2010).

[12] S. Takei and Y. Tserkovnyak, Superfluid Spin Transport Through Easy-Plane Ferromagnetic Insulators, Phys. Rev. Lett. 112, 227201 (2014).

[13] R. A. Duine, A. Brataas, S. A. Bender, and Y. Tserkovnyak, Spintronics and magnon Bose-Einstein condensation, arXiv:1505.01329.

[14] L. J. Cornelissen, J. Liu, R. A. Duine, J. Ben Youssef, and B. J. van Wees, Long-distance transport of magnon spin information in a magnetic insulator at room temperature, Nat. Phys. 11, 1022 (2015).

[15] A. V. Chumak, V. I. Vasyuchka, A. A. Serga, and B. Hillebrands, Magnon spintronics, Nat. Phys. 11, 453 (2015).

[16] K. W. Madison, F. Chevy, W. Wohlleben, and J. Dalibard, Vortex Formation in a Stirred Bose-Einstein Condensate, Phys. Rev. Lett. 84, 806 (2000).

[17] O. M. Maragò, S. A. Hopkins, J. Arlt, E. Hodby, G. Hechenblaikner, and C. J. Foot, Observation of the Scissors Mode and Evidence for Superfluidity of a Trapped Bose-Einstein Condensed Gas, Phys. Rev. Lett. 84, 2056 (2000).

[18] J. R. Abo-Shaeer, C. Raman, J. M. Vogels, and W. Ketterle, Observation of vortex lattices in Bose-Einstein condensates, Science 292, 476 (2001).

[19] D. M. Stamper-Kurn, M. R. Andrews, A. P. Chikkatur, S. Inouye, H.-J. Miesner, J. Stenger, and W. Ketterle, Optical Confinement of a Bose-Einstein Condensate, Phys. Rev. Lett. 80, 2027 (1998).

[20] J. Stenger, S. Inouye, D. M. Stamper-Kurn, H.-J. Miesner, A. P. Chikkatur, and W. Ketterle, Spin domains in ground-state BoseEinstein condensates, Nature (London) 396, 345 (1998). 
[21] L. E. Sadler, J. M. Higbie, S. R. Leslie, M. Vengalattore, and D. M. Stamper-Kurn, Spontaneous symmetry breaking in a quenched ferromagnetic spinor Bose-Einstein condensate, Nature (London) 443, 312 (2006).

[22] J.-y. Choi, S. Kang, S. W. Seo, W. J. Kwon, and Y.-i. Shin, Observation of a Geometric Hall Effect in a Spinor BoseEinstein Condensate with a Skyrmion Spin Texture, Phys. Rev. Lett. 111, 245301 (2013).

[23] G. E. Marti, A. MacRae, R. Olf, S. Lourette, F. Fang, and D. M. Stamper-Kurn, Coherent Magnon Optics in a Ferromagnetic Spinor Bose-Einstein Condensate, Phys. Rev. Lett. 113, 155302 (2014).

[24] R. W. Cherng, V. Gritsev, D. M. Stamper-Kurn, and E. Demler, Dynamical Instability of the $X Y$ Spiral State of Ferromagnetic Condensates, Phys. Rev. Lett. 100, 180404 (2008).

[25] V. I. Yukalov, Difference in Bose-Einstein condensation of conserved and unconserved particles, Laser Phys. 22, 1145 (2012).

[26] F. Fang, R. Olf, S. Wu, H. Kadau, and D. M. Stamper-Kurn, Condensing Magnons in a Degenerate Ferromagnetic Spinor Bose Gas, Phys. Rev. Lett. 116, 095301 (2016).

[27] S. Ashhab, Superfluid vs ferromagnetic behavior in a Bose gas of spin-1/2 atoms, J. Low Temp. Phys. 140, 51 (2005).

[28] K. Kudo and Y. Kawaguchi, Dissipative hydrodynamic equation of a ferromagnetic Bose-Einstein condensate: Analogy to magnetization dynamics in conducting ferromagnets, Phys. Rev. A 84, 043607 (2011).

[29] H. Flayac, H. Terças, D. D. Solnyshkov, and G. Malpuech, Superfluidity of spinor Bose-Einstein condensates, Phys. Rev. B 88, 184503 (2013).

[30] Q. Zhu, Q.-f. Sun, and B. Wu, Superfluidity of a pure spin current in ultracold Bose gases, Phys. Rev. A 91, 023633 (2015).

[31] Y. M. Bunkov and G. E. Volovik, Magnon Bose-Einstein condensation and spin superfluidity, J. Phys.: Condens. Matter 22, 164210 (2010).

[32] E. Yukawa and M. Ueda, Hydrodynamic description of spin-1 Bose-Einstein condensates, Phys. Rev. A 86, 063614 (2012).

[33] D. M. Stamper-Kurn and M. Ueda, Spinor Bose gases: Symmetries, magnetism, and quantum dynamics, Rev. Mod. Phys. 85, 1191 (2013).

[34] M. Ueda, Bose gases with nonzero spin, Annu. Rev. Condens. Matter Phys. 3, 263 (2012).

[35] We concentrate on the regime where $p>0$ and $K>0$ in this paper; see also Sec. III A.

[36] K. Murata, H. Saito, and M. Ueda, Broken-axisymmetry phase of a spin-1 ferromagnetic Bose-Einstein condensate, Phys. Rev. A 75, 013607 (2007).

[37] N. T. Phuc, Y. Kawaguchi, and M. Ueda, Effects of thermal and quantum fluctuations on the phase diagram of a spin- $1{ }^{87} \mathrm{Rb}$ Bose-Einstein condensate, Phys. Rev. A 84, 043645 (2011).

[38] T. Ohmi and K. Machida, Bose-Einstein condensation with internal degrees of freedom in alkali atom gases, J. Phys. Soc. Jpn. 67, 1822 (1998).

[39] T.-L. Ho, Spinor Bose Condensates in Optical Traps, Phys. Rev. Lett. 81, 742 (1998).

[40] Y.-T. Oh, P. Kim, J.-H. Park, and J. H. Han, Manifold Mixing in the Temporal Evolution of a Spin-1 Spinor Bose-Einstein Condensate, Phys. Rev. Lett. 112, 160402 (2014).

[41] A. Lamacraft, Long-wavelength spin dynamics of ferromagnetic condensates, Phys. Rev. A 77, 063622 (2008).
[42] M. Kunimi and H. Saito, Upper bound of one-magnon excitation and lower bound of effective mass for ferromagnetic spinor Bose and Fermi gases, Phys. Rev. A 91, 043624 (2015).

[43] J. Armaitis, H. T. C. Stoof, and R. A. Duine, Magnetization Relaxation and Geometric Forces in a Bose Ferromagnet, Phys. Rev. Lett. 110, 260404 (2013).

[44] T. L. Gilbert, A phenomenological theory of damping in ferromagnetic materials, IEEE Trans. Magn. 40, 3443 (2004).

[45] R. Olf, F. Fang, G. E. Marti, A. MacRae, and D. M. StamperKurn, Thermometry and cooling of a Bose gas to 0.02 times the condensation temperature, Nat. Phys. 11, 720 (2015).

[46] Y. Kawaguchi, H. Saito, and M. Ueda, Can Spinor Dipolar Effects be Observed in Bose-Einstein Condensates? Phys. Rev. Lett. 98, 110406 (2007).

[47] T. Holstein and H. Primakoff, Field dependence of the intrinsic domain magnetization of a ferromagnet, Phys. Rev. 58, 1098 (1940).

[48] A. Auerbach, Interacting Electrons and Quantum Magnetism (Springer-Verlag, Berlin, 1994).

[49] S. O. Demokritov, V. E. Demidov, O. Dzyapko, G. A. Melkov, A. A. Serga, B. Hillebrands, and A. N. Slavin, Bose-Einstein condensation of quasi-equilibrium magnons at room temperature under pumping, Nature (London) 443, 430 (2006).

[50] R. E. Troncoso and Á. S. Núñez, Josephson effects in a BoseEinstein condensate of magnons, Ann. Phys. (N.Y.) 346, 182 (2014).

[51] Q. Gu, K. Bongs, and K. Sengstock, Spin waves in ferromagnetically coupled spinor Bose gases, Phys. Rev. A 70, 063609 (2004)

[52] Q. Gu and R. A. Klemm, Ferromagnetic phase transition and Bose-Einstein condensation in spinor Bose gases, Phys. Rev. A 68, 031604 (2003).

[53] D. S. Hall, M. R. Matthews, J. R. Ensher, C. E. Wieman, and E. A. Cornell, Dynamics of Component Separation in a Binary Mixture of Bose-Einstein Condensates, Phys. Rev. Lett. 81, 1539 (1998).

[54] B. Flebus, S. A. Bender, Y. Tserkovnyak, and R. A. Duine, Two-Fluid Theory for Spin Superfluidity in Magnetic Insulators, Phys. Rev. Lett. 116, 117201 (2016).

[55] H. T. C. Stoof, Coherent versus incoherent dynamics during Bose-Einstein condensation in atomic gases, J. Low Temp. Phys. 114, 11 (1999).

[56] T. Giamarchi, Ch. Rüegg, and O. Tchernyshyov, BoseEinstein condensation in magnetic insulators, Nat. Phys. 4, 198 (2008).

[57] C. Pfleiderer, Superconducting phases of $f$-electron compounds, Rev. Mod. Phys. 81, 1551 (2009).

[58] B. I. Halperin and P. C. Hohenberg, Hydrodynamic theory of spin waves, Phys. Rev. 188, 898 (1969).

[59] L. Landau, Theory of the superfluidity of helium II, Phys. Rev. 60, 356 (1941).

[60] Y. Kawaguchi and M. Ueda, Spinor Bose Einstein condensates, Phys. Rep. 520, 253 (2012).

[61] A. L. Gaunt, T. F. Schmidutz, I. Gotlibovych, R. P. Smith, and Z. Hadzibabic, Bose-Einstein Condensation of Atoms in a Uniform Potential, Phys. Rev. Lett. 110, 200406 (2013).

[62] L. Corman, L. Chomaz, T. Bienaimé, R. Desbuquois, C. Weitenberg, S. Nascimbène, J. Dalibard, and J. Beugnon, Quench-Induced Supercurrents in an Annular Bose Gas, Phys. Rev. Lett. 113, 135302 (2014). 
[63] D. Niroomand, S. D. Graham, and J. M. McGuirk, Longitudinal Spin Diffusion in a Nondegenerate Trapped ${ }^{87} \mathrm{Rb}$ Gas, Phys. Rev. Lett. 115, 075302 (2015).

[64] L. Pitaevskii and S. Stringari, Bose-Einstein Condensation and Superfluidity (Oxford University Press, Oxford, 2016), Vol. 164.

[65] F. J. Dyson, General theory of spin-wave interactions, Phys. Rev. 102, 1217 (1956).

[66] F. Keffer and R. Loudon, Simple physical theory of spin wave interactions, J. Appl. Phys. 32, S2 (1961).

[67] A. B. Harris, Energy width of spin waves in the Heisenberg ferromagnet, Phys. Rev. 175, 674 (1968).

[68] J. Van Kranendonk and J. H. Van Vleck, Spin waves, Rev. Mod. Phys. 30, 1 (1958).

[69] A. I. Akhiezer, V. G. Bar'yakhtar, and S. V. Peletminskii, On the theory of relaxation processes in ferrodielectrics at low temperatures, JETP 9, 146 (1959).

[70] H.-J. Miesner, D. M. Stamper-Kurn, M. R. Andrews, D. S. Durfee, S. Inouye, and W. Ketterle, Bosonic stimulation in the formation of a Bose-Einstein condensate, Science 279, 1005 (1998).

[71] A. J. Leggett, Bose-Einstein condensation in the alkali gases: Some fundamental concepts, Rev. Mod. Phys. 73, 307 (2001).
[72] R. Meppelink, R. van Rooij, J. M. Vogels, and P. van der Straten, Enhanced Heat Flow in the Hydrodynamic Collisionless Regime, Phys. Rev. Lett. 103, 095301 (2009).

[73] For a homogeneous ${ }^{87} \mathrm{Rb}$ gas comparable to the experiments in Refs. [23,45] with a density corresponding to the condensation temperature of $1 \mu \mathrm{K}$, at the temperature of $0.1 \mu \mathrm{K}$, the collision time of atoms is $0.6 \mathrm{~ms}$.

[74] J. Armaitis, H. T. C. Stoof, and R. A. Duine, Hydrodynamic modes of partially condensed Bose mixtures, Phys. Rev. A 91, 043641 (2015).

[75] S. B. Koller, A. Groot, P. C. Bons, R. A. Duine, H. T. C. Stoof, and P. van der Straten, Quantum enhancement of spin drag in a Bose gas, New J. Phys. 17, 113026 (2015).

[76] T. Lahaye, C. Menotti, L. Santos, M. Lewenstein, and T. Pfau, The physics of dipolar bosonic quantum gases, Rep. Prog. Phys. 72, 126401 (2009).

[77] C. Chin, R. Grimm, P. Julienne, and E. Tiesinga, Feshbach resonances in ultracold gases, Rev. Mod. Phys. 82, 1225 (2010).

[78] D. Vollhardt and P. Wolfle, The Superfluid Phases of Helium 3 (Dover, New York, 2013).

[79] J. Linder and J. W. A. Robinson, Superconducting spintronics, Nat. Phys. 11, 307 (2015). 